\title{
Effets du trempage des semences sur le rendement du sorgho dans les conditions du Sahel à Kolokani et Diéma au Mali.
}

\author{
ZEROME Moussa1, TRAORE Kalifa², FAMANTA Mahamoudou³, SAMAKE Oumar2, TOGO Moise \\ Anewin 4 \\ 1'Institut d'Economie Rurale (IER), Centre Régional de Recherche Agronomique de Mopti, BP : 205, Mopti, Mali. \\ Téléphone : (+223) 76044013 / 653092 29. E-mail : zeromemoussa@yahoo.fr \\ 2Institut d'Economie Rurale (IER), Centre Régional de Recherche Agronomique (CRRA) de Sotuba, Laboratoire Sol- \\ Eau-Plante BP: 262, Bamako, Mali. E-mail: ibosimon_1@yahoo.fr \\ 3Institut Polytechnique de Formation et de Recherche Appliquée de Katibougou, BP : 06, Koulikoro, Mali. \\ 4World Vision, Badalabougou (face au palais de la culture), Rue 56 Porte 403, BP 2347, Bamako, Mali. \\ Correspondant : *Moussa ZEROME, E-mail : zeromemoussa@yahoo.fr, Tel. (+223) 76044013 / 65309229.
}

Original submitted in on $18^{\text {th }}$ January 2019. Published online at www.m.elewa.org/journals/ on $30^{\text {th }}$ April 2019 https://dx.doi.org/10.4314/jab.v136i1.5

\section{RESUME}

Objectif: L'objectif de l'étude était d'évaluer l'effet du trempage sous différentes fertilisations sur le rendement du sorgho.

Méthodologie et résultats: Des échantillons de sol ont été prélevés pour des analyses physico-chimiques au laboratoire afin de déterminer la fertilité initiale des parcelles d'expérimentations. Les essais ont été installés sur une superficie totale de 20 hectares dans les villages de Tiéneguebougou, de Farako, de Guémou et de Bignekolobougou. Le dispositif expérimental était le split plot avec 20 répétitions. Les facteurs étudiés étaient la technologie du trempage des semences conseillées par la recherche et la pratique paysanne (témoin) du trempage des semences avec 4 niveaux de fertilisation. Pour la technologie du trempage des semences, le principe consistait à faire tremper les graines dans l'eau ordinaire pendant 8 heures, suivi d'un séchage à l'ombre pendant 2 heures avant le semis. Le témoin était la pratique paysanne habituelle de la zone dont la durée du trempage était d'environ 5 heures et le séchage se faisait au soleil. Les semences ont été prétraitées à l'apron star et le semis a été réalisé en lignes sur des billons avec un écartement de $80 \mathrm{~cm} \times 40 \mathrm{~cm}$. La fumure organique (100g/poquet) et le NPK 17-17-17 (6g/poquet) ont été apportés en micro-dose au moment du semis. L'urée $(46 \% \mathrm{~N})$ a été enfouie en micro-dose $(1 \mathrm{~g}$ soit une pincée à trois doigts) à côté des plants de sorgho en début de la montaison après une pluie assurant un sol humide. Les résultats des analyses ont montré que les sols présentent une texture sablo-limoneuse à limono-sableuse avec une faible fertilité, une acidité, une carence en phosphore, en azote et en potassium. Le trempage des semences a produit des rendements grain de $731 \mathrm{~kg} / \mathrm{ha}, 1858 \mathrm{~kg} / \mathrm{ha}$ de paille, 33342 panicules/ha, $1051 \mathrm{~kg} / \mathrm{ha}$ de poids panicules. Concernant le témoin, les rendements étaient de $202 \mathrm{~kg} / \mathrm{ha}$ de grain, $482 \mathrm{~kg} / \mathrm{ha}$ de paille, 15592 panicules/ha et $313 \mathrm{~kg} / \mathrm{ha}$ de poids panicules. L'apport du fumier et de l'engrais minéral en micro-dose a augmenté significativement le rendement grain de $33 \%$ et le rendement paille de $54 \%$ par rapport au témoin. Le trempage a permis d'augmenter le nombre de 
panicules de plus $100 \%$; les rendements grain, paille et poids panicules de plus $200 \%$ comparativement au témoin.

Conclusion et application des résultats: Au regard des résultats probants obtenus, la technologie du trempage des semences a permis de doubler voire tripler le rendement du sorgho par rapport au témoin. La technologie du trempage des semences est applicable partout au Sahel et dans d'autres zones à écologie similaire.

Mots-clés : trempage, fertilisation, rendement, Mali.

\section{Effects of seed soaking on sorghum yield in Sahelian zone of Kolokani and Diema in Mali. ABSTRACT}

Objective: The objective of the study was to assess the effect of seed soaking under different fertilizations on sorghum yield.

Methodology and results: Soil samples were taken for physico-chemical analyzes in the laboratory to determine the initial fertility of the experimental plots. The tests were implemented on a total area of 20 hectares in the villages of Tieneguebougou, Farako, Guemou and Bignekolobougou. The experimental device was the split plot with 20 repetitions. The factors studied were the seed soaking technology recommended by research and seed soaking according to farmer practice (control) with 4 levels of fertilization. For the seed soaking technology, the principle was to soak the seeds in ordinary water for 8 hours, followed by drying in the shade for 2 hours before sowing. The control was the usual farmer practice in the area, whose soaking time was about 5 hours and seed drying had done in the sun. The seeds were pre-treated with apron star and sowing was done in rows on ridges with a spacing of $80 \mathrm{~cm} \mathrm{x} 40 \mathrm{~cm}$. Organic manure $(100 \mathrm{~g} / \mathrm{hole})$ and NPK 17-17-17 (6g/hole) were provided in micro-dose at the time of sowing. Urea $(46 \% \mathrm{~N})$ was brought in a micro-dose $(1 \mathrm{~g} /$ hole either a three-finger pinch) next to sorghum seedlings at the beginning of elongation after a rainfall ensuring moist soil. Results showed that soils had sandy-loamy and sandy-loamy texture with low fertility, acidity and deficiency in phosphorus, nitrogen and potassium. The experiment was laid in split plot design with 20 repetitions. Seed soaking technology provided $731 \mathrm{~kg} \mathrm{ha}^{-1}$ of grain yield, $1858 \mathrm{~kg} \mathrm{ha}^{-1}$ of straw, 33342 panicles ha-1, $1051 \mathrm{~kg} \mathrm{ha}^{-1}$ of panicle weight. For traditional practice (control), yields were $202 \mathrm{~kg}^{-1}$ of grain, $482 \mathrm{~kg} \mathrm{ha}^{-1}$ of straw, 15592 panicles ha-1 and $313 \mathrm{~kg} \mathrm{ha}^{-1}$ of panicle weight. Micro dose application of manure and mineral fertilizer increased significantly the grain yield by $33 \%$ and straw yield by $54 \%$ compared to the control. Seed soaking increased panicle number by over $100 \%$ and more than $200 \%$ for grain, straw and panicle yields comparatively to the control.

Conclusion and application of results: According to the potent results, seed soaking technology has permitted to double either triple sorghum yield comparatively to the control. Seed soaking technology could be applied as well in the Sahel as elsewhere similar to the Sahel condition.

Keywords: soaking, fertilization, yield, Mali

\section{INTRODUCTION}

Dans les zones arides et semi-arides d'Afrique subsaharienne, la production céréalière est largement tributaire de la variabilité pluviométrique entrainant une faible densité des cultures (Jones et Wanbi, 1992 ; Bougne et al., 2000 ; Biasutti et al., 2009 ; Damirkaya et al., 2006 ; Dingkuhn et al., 2006). De plus, les mauvaises herbes entrent en concurrence avec les cultures pour la lumière, l'eau et les nutriments (Kropff et Van Laar, 1993).
Les plants sains avec des systèmes radiculaires bien développés peuvent mieux résister aux conditions défavorables, présentent une bonne vigueur à la levée et produisent des rendements plus élevés (Harris et al., 2000). La bonne densité des cultures est une condition préalable à l'utilisation efficace des ressources telles que l'eau et la lumière pour l'obtention de meilleur rendement. Selon la même source, la germination 
et la croissance rapide des cultures sont des facteurs déterminants des rendements plus élevés. Une des méthodes pour améliorer l'établissement des cultures consiste à faire le trempage des semences qui améliore la germination et augmente la vigueur des plants (Harris, 1999). Les techniques de trempage des semences, de conservation de l'eau, et de fertilisation ont fait leur preuve comme mesures d'adaptation des cultures au changement climatique en Asie, en Afrique de l'Est notamment en Ethiopie, au Soudan et en Erythrée, pays durement touchés par la sécheresse. Les effets bénéfiques immédiats $d u$ trempage des semences sont multiples : le raccourcissement de la durée de germination, la bonne densité de semis, la vigueur des plants et

\section{MATERIEL ET METHODES}

Localisation des sites d'expérimentation: Les essais ont été conduits dans les communes rurales de Kolokani (-8.036540 longitude Ouest et 13.574579 latitude Nord), Didiéni (-8.087920 longitude Ouest et 13.881109 latitude Nord), Diéma (-9.188129 longitude Ouest et 14.543499 latitude Nord) et DiankountéCamara (-9.515750 longitude Ouest et 14.543820 latitude Nord) au Mali. Le climat est de type soudanosahélien avec une pluviométrie de 500 à $600 \mathrm{~mm} / \mathrm{an}$. La saison sèche se caractérise par deux périodes, une période froide allant de mi-novembre à mi-mars avec des températures minimales de $12^{\circ} \mathrm{C}$ à $14^{\circ} \mathrm{C}$ et une l'augmentation de la production (Harris, 2001; Coulibaly et al., 2012). Les expérimentations réalisées au Mali, au Soudan et en Éthiopie ont démontré que le trempage augmente le rendement des cultures d'environ 30\% (Harris, 2000; Coulibaly et al., 2012; Harris et al., 2001). Les mêmes auteurs affirment que le rendement obtenu avec le trempage des semences peut souvent doubler celui obtenu avec la pratique paysanne. Toutefois peu d'études existent sur la combinaison de la technique de microdosage des engrais et le trempage des semences en zone sahélienne du Mali. L'objectif de la présente étude était d'évaluer l'effet du trempage associé à l'application en micro doses des engrais minéraux et organiques sur les rendements du sorgho.

période chaude (mars -juin) avec des températures variant entre $39^{\circ} \mathrm{C}$ et $43^{\circ} \mathrm{C}$. La moyenne annuelle de I'ETP varie de $1800 \mathrm{~mm}$ au Nord à $1700 \mathrm{~mm}$ au Sud. Cette moyenne élevée de l'évapotranspiration dans le nord explique la déshydratation des plantes et l'appartenance de la zone au domaine sahélien. La végétation est composée majoritairement de Piliostigma reticulatum, Combretum glutinosum, Guiera senegalensis, Cassia sieberiana et Acacia nilotica. La figure 1 montre la situation géographique des sites d'étude. 


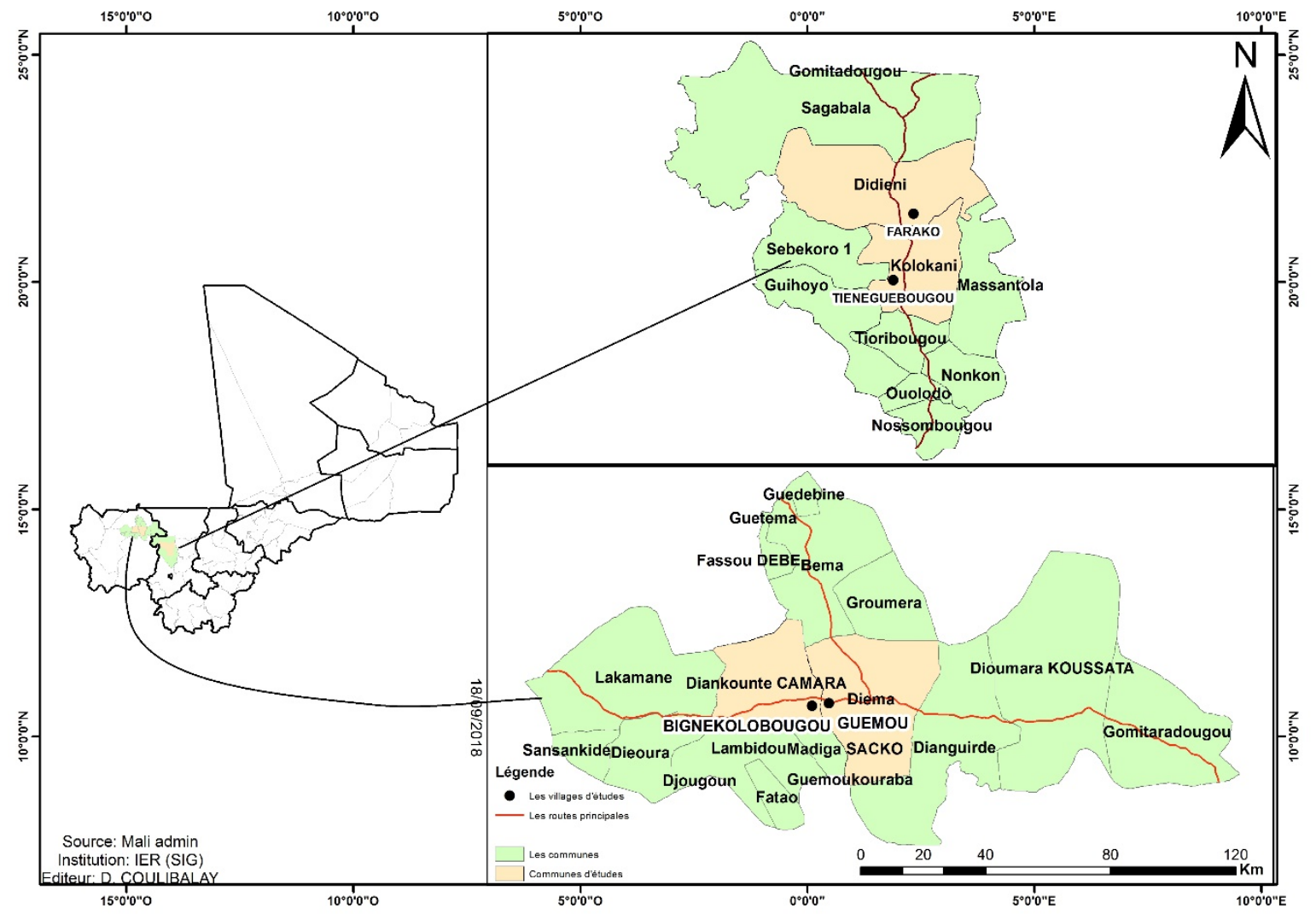

Figure 1 : Localisation des sites d'étude

Caractérisation physico-chimique des sols et cartographie des parcelles expérimentales : II a été procédé au prélèvement d'échantillons de sol dans les parcelles d'essai à deux niveaux $(0-10$ et $10-20 \mathrm{~cm})$ de profondeur. L'analyse des propriétés physico-chimiques a concerné l'azote, le phosphore, le potassium, le carbone, le pH (eau), la CEC et les bases échangeables $(\mathrm{Ca}, \mathrm{Mg}, \mathrm{Na})$. La méthode potentiométrique a été utilisée pour le dosage du $\mathrm{pH}$ (eau); la méthode Anne modifiée pour la matière organique $(\% \mathrm{C})$; la méthode de colorimétrie pour l'azote total $(\% \mathrm{~N})$; la méthode Bray II pour le phosphore assimilable (ppm) ; et la méthode METSON à partir d'un dosage à l'Acétate d'ammonium pour la mesure de la CEC (meq/100g). La cartographie des parcelles d'essai a été réalisée au moyen du ruban pour fixer les 4 sommets de chaque parcelle et le GPS (Global Positioning System) pour le géo-référencement des pourtours et des ligneux qui s'y trouvaient. Ensuite, les coordonnées géographiques ont été transférées dans le logiciel « Arc gis » pour l'élaboration des cartes des parcelles expérimentales.

Installation des essais : Les essais ont été installés sur une superficie totale de 20 hectares dans les villages de Tiéneguebougou, Farako, Guémou et de Bignekolobougou soit 5 ha par village. Les traitements étaient les suivants :

T1 : culture du sorgho sans apport de fertilisants

T2 : culture du sorgho + micro-dose de fumier

T3 : culture du sorgho + micro-dose d'engrais minéral

T4 : culture du sorgho + micro-dose fumier + microdose engrais minéral.

Le dispositif expérimental était le split plot avec vingt (20) répétitions. Les facteurs étudiés étaient la technologie du trempage des semences conseillées par la recherche et la pratique paysanne (témoin) du trempage des semences avec les 4 niveaux de fertilisation ci-dessus indiqués. La dimension d'une parcelle élémentaire était de $150 \mathrm{~m}^{2}(30 \mathrm{~m} \times 5 \mathrm{~m})$. La distance inter-parcellaire était de $1 \mathrm{~m}$. Celle des interblocs était de $2 \mathrm{~m}$. Pour le trempage des semences, il s'agissait d'évaluer l'effet du trempage sous différentes fertilisations sur le rendement du sorgho. Le principe consistait à faire tremper les graines dans l'eau ordinaire pendant 8 heures, suivi d'un séchage à l'ombre pendant 2 heures avant le semis. Le témoin était la pratique paysanne habituelle de la zone dont la durée du trempage était d'environ 5 heures et le 
séchage se faisait au soleil. Les semences ont été prétraitées à l'apron star et le semis a été réalisé en lignes sur des billons avec un écartement de $80 \mathrm{~cm} \mathrm{x}$ $40 \mathrm{~cm}$ dans les parcelles expérimentales. La fumure organique (100g/poquet) et le NPK 17-17-17 (6g/poquet) ont été apportés en micro-dose au moment du semis. L'urée ( $46 \% \mathrm{~N}$ ) a été enfouie en micro-dose (1 g soit une pincée à trois doigts) à côté des plants de sorgho en début de la montaison après une pluie assurant un sol humide.

\section{RESULTATS}

Caractérisation physico-chimique des sols et cartographie des parcelles expérimentales: Les figures 2 et 3 montrent les points de prélèvement des
Données collectées: Les variables mesurées ont porté sur la caractérisation physico-chimique des sols et l'évaluation des rendements du sorgho par la collecte du poids de la paille; le poids des panicules pleines; les grains et le nombre de panicules récoltées.

Analyse des données: Les données collectées ont été saisies dans Excel puis analysées avec le logiciel d'analyse statistique GenStat 12.1 en utilisant la méthode d'analyse de variance et le classement des moyennes avec le test de la ppds au seuil de $5 \%$.

échantillons de sol dans les parcelles d'essai dans les localités de Kolokani et Diéma.

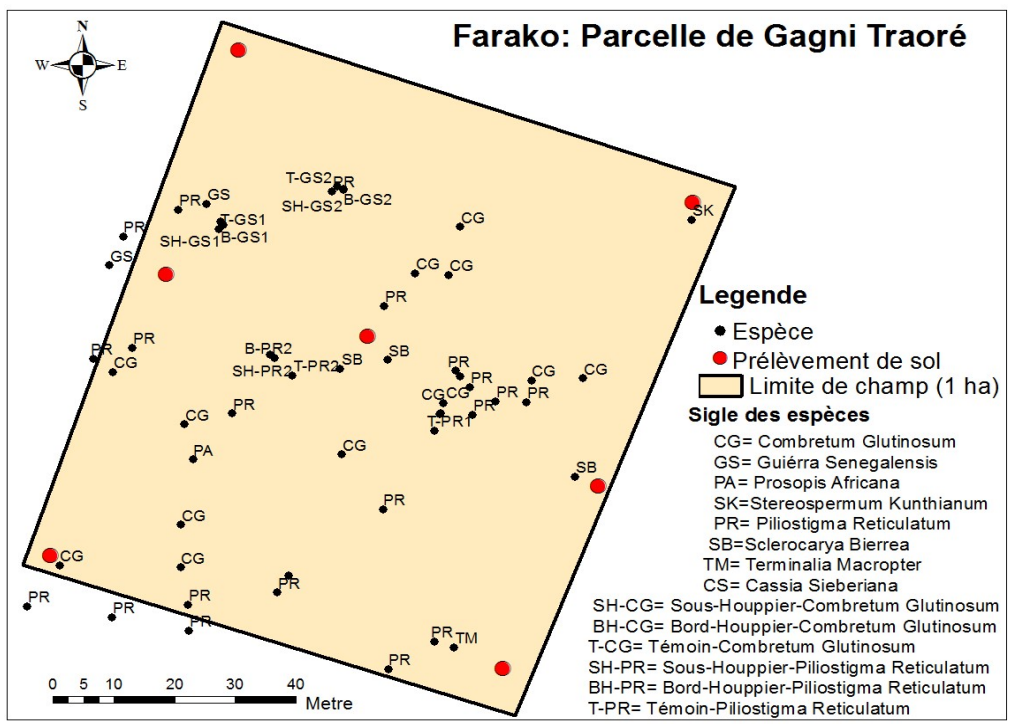

Figure 2 : Carte de la parcelle expérimentale de Gagni Traoré à Farako (Kolokani) au Mali

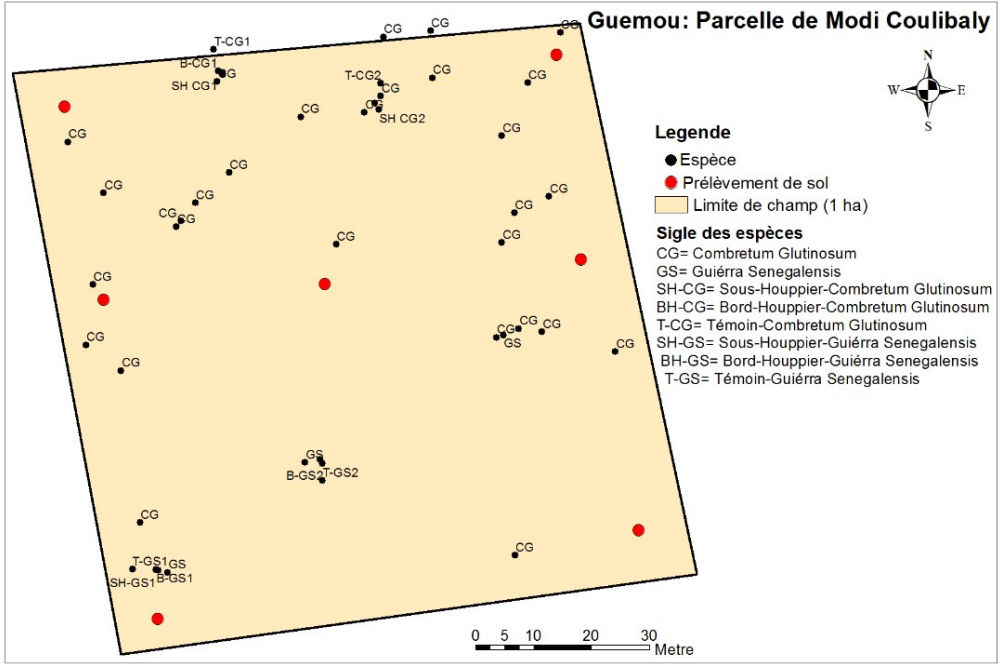

Figure 3 : Carte de la parcelle d'essai de Mody Coulibaly à Guémou (Diéma) au Mali 
Tableau 1: Caractérisation physico-chimique des sols de Kolokani

\begin{tabular}{|c|c|c|c|}
\hline \multirow[t]{2}{*}{ Paysans } & \multirow[t]{2}{*}{ Analyse réalisée } & \multicolumn{2}{|c|}{ Profondeur } \\
\hline & & $0-10 \mathrm{~cm}$ & $10-20 \mathrm{~cm}$ \\
\hline \multirow[t]{13}{*}{ Madou Diarra } & $\mathrm{pH}$ & 6.21 & 6.33 \\
\hline & M.O & 1.45 & 1.65 \\
\hline & $\mathrm{N}$ & 0.01 & 0.01 \\
\hline & $P$ & 4.14 & 7.77 \\
\hline & CEC & 4.15 & 4.79 \\
\hline & $\mathrm{Ca}$ & 1.64 & 2.20 \\
\hline & $\mathrm{Mg}$ & 0.77 & 0.87 \\
\hline & K & 0.23 & 0.21 \\
\hline & $\mathrm{Na}$ & 0.01 & 0.01 \\
\hline & Sable & 69 & 60 \\
\hline & Limon & 29 & 38 \\
\hline & Argile & 2 & 2 \\
\hline & C.T & LS & LS \\
\hline \multirow[t]{13}{*}{ Naba Diarra } & $\mathrm{pH}$ & 5.43 & 5.71 \\
\hline & M.O & 0.11 & 0.34 \\
\hline & $\mathrm{N}$ & 0.01 & 0.01 \\
\hline & $P$ & 0.97 & 1.21 \\
\hline & CEC & 3.55 & 3.87 \\
\hline & $\mathrm{Ca}$ & 1.18 & 1.37 \\
\hline & $\mathrm{Mg}$ & 0.66 & 0.74 \\
\hline & K & 0.21 & 0.26 \\
\hline & $\mathrm{Na}$ & - & - \\
\hline & Sable & 76 & 75 \\
\hline & Limon & 22 & 23 \\
\hline & Argile & 2 & 2 \\
\hline & C.T & SL & SL \\
\hline
\end{tabular}

Légende : M.O = matière organique $; \mathrm{C} . \mathrm{T}=$ classe= classe texturale $; \mathrm{CEC}=$ capacité d'échange cationique ; =LS=LimonoSableux ; SL=Sablo-Limoneux

L'analyse des échantillons de sol a montré que les sites des essais ont une texture sablo-limoneuse à limonosableuse (tableaux 1 et 2). Les sols sont acides avec des valeurs de $\mathrm{pH}$ eau oscillant entre 5,43 et 5,71 (tableau 1). Le taux de matière organique est faible dans les sols avec des valeurs comprises entre 0,11 et 0,34 . Les sols sont aussi pauvres en azote et en phosphore où les taux varient de 0,01 à $0,01 \%$ et de 0,97 à 1,21 ppm respectivement (tableau 1). La réserve en éléments nutritifs exprimée par la CEC est moyenne (4,5 à 4,01) dans les sols où les teneurs en calcium $(0,92$ à 0,60$)$, magnésium $(0,44$ à 0,51$)$ et potassium $(0,25$ à 0,24$)$ sont très faibles (tableau 2). Le taux de sodium échangeable est très faible (tableaux 1 et 2). 
Tableau 2 : Caractérisation physico-chimique des sols de Diéma

\begin{tabular}{|c|c|c|c|}
\hline \multirow[t]{2}{*}{ Paysans } & \multirow[t]{2}{*}{ Analyse réalisée } & \multicolumn{2}{|c|}{ Profondeur } \\
\hline & & $0-10 \mathrm{~cm}$ & $10-20 \mathrm{~cm}$ \\
\hline \multirow[t]{13}{*}{ Waradjougou Coulibaly } & $\mathrm{pH}$ & 5.66 & 6.31 \\
\hline & M.O & 0.25 & 0.13 \\
\hline & $\mathrm{N}$ & 0.01 & 0.01 \\
\hline & $P$ & 1.21 & 1.35 \\
\hline & CEC & 3.14 & 2.98 \\
\hline & $\mathrm{Ca}$ & 0.92 & 0.60 \\
\hline & $\mathrm{Mg}$ & 0.44 & 0.51 \\
\hline & K & 0.25 & 0.35 \\
\hline & $\mathrm{Na}$ & 0.03 & 0.02 \\
\hline & Sable & 88 & 90 \\
\hline & Limon & 8 & 8 \\
\hline & Argile & 4 & 2 \\
\hline & C.T & SL & SL \\
\hline \multirow{13}{*}{ Balla Traoré } & $\mathrm{pH}$ & 5.84 & 5.92 \\
\hline & M.O & 0.13 & 0.18 \\
\hline & $\mathrm{N}$ & 0.01 & 0.02 \\
\hline & $P$ & 4.22 & 5.38 \\
\hline & CEC & 4.50 & 4.01 \\
\hline & $\mathrm{Ca}$ & 1.90 & 1.39 \\
\hline & $\mathrm{Mg}$ & 0.80 & 0.83 \\
\hline & K & 0.25 & 0.24 \\
\hline & $\mathrm{Na}$ & 0.05 & 0.05 \\
\hline & Sable & 90 & 82 \\
\hline & Limon & 8 & 16 \\
\hline & Argile & 2 & 2 \\
\hline & C.T & SL & SL \\
\hline
\end{tabular}

Légende : M.O = matière organique $; \mathrm{C} . \mathrm{T}=$ classe texturale $; \mathrm{CEC}=$ capacité d'échange cationique $; \mathrm{SL}=$ SabloLimoneux

Effet de la technologie du trempage des semences sur les rendements du sorgho: Les rendements obtenus avec la technologie du trempage des semences étaient plus élevés que ceux de la pratique paysanne de trempage des semences (témoin). La technologie du trempage des semences a donné 731 $\mathrm{kg} / \mathrm{ha}$ de rendement grain, $1858 \mathrm{~kg} / \mathrm{ha}$ de paille,
33342 panicules/ha et $1051 \mathrm{~kg} / \mathrm{ha}$ de poids panicules (tableau 3). Pour la pratique paysanne, il a été obtenu $202 \mathrm{~kg} / \mathrm{ha}$ de grain, $482 \mathrm{~kg} / \mathrm{ha}$ de paille, 15592 panicules/ha et $313 \mathrm{~kg} / \mathrm{ha}$ de poids panicules. Les poids de 1000 graines étaient respectivement $24 \mathrm{~g}$ pour la technologie trempage des semences et $19 \mathrm{~g}$ pour le témoin.

Tableau 3 : Effet de la technologie du trempage des semences sur les rendements du sorgho

\begin{tabular}{l|c|c|c|c|c}
\hline Technologies & $\begin{array}{l}\text { Rendement } \\
\text { grain (kg/ha) }\end{array}$ & $\begin{array}{l}\text { Rendement } \\
\text { paille (kg/ha) }\end{array}$ & $\begin{array}{l}\text { Nombre de } \\
\text { panicules } \\
\text { (nombre/ha) }\end{array}$ & $\begin{array}{l}\text { Rendement } \\
\text { panicules } \\
\text { (kg/ha) }\end{array}$ & $\begin{array}{l}\text { Poids de } \\
\mathbf{1 0 0 0}(\mathbf{g})\end{array}$ \\
\hline Situation & $731 \mathrm{a}$ & $1858 \mathrm{a}$ & $33342 \mathrm{a}$ & $1051 \mathrm{a}$ & $24,21 \mathrm{a}$ \\
Trp & $202 \mathrm{~b}$ & $482 \mathrm{~b}$ & $15592 \mathrm{~b}$ & $313 \mathrm{~b}$ & $19,31 \mathrm{a}$ \\
TTrp & $<0,001$ & $<0,001$ & $<0,001$ & $<0,001$ & 0,84 \\
Probabilité & $\mathrm{HS}$ & $\mathrm{HS}$ & $\mathrm{HS}$ & $\mathrm{HS}$ & $\mathrm{NS}$ \\
Signification & 21,6 & 34,21 & 156,42 & 26,12 & 4,66 \\
\hline Ecart-type & & & & \\
\hline
\end{tabular}

Légende : Trp = trempage $;$ T Trp = témoin du trempage 
L'application de la fertilisation organo-minérale a augmenté les rendements du sorgho local. Elle a produit $889,1 \mathrm{~kg} / \mathrm{ha}$ de rendement grain contre 111,9 $\mathrm{kg} / \mathrm{ha}$ pour le témoin (tableau 4). Pour la paille, le rendement était de $2470 \mathrm{~kg} / \mathrm{ha}$ contre $178 \mathrm{~kg} / \mathrm{ha}$ pour le témoin. L'apport du fumier et du NPK a donné 28,06 $\mathrm{g}$ de poids de 1000 graines contre 17,94 g pour le témoin.

Tableau 4 : Effet de la technologie du trempage des semences avec différentes doses de fertilisation sur les rendements du sorgho

\begin{tabular}{|c|c|c|c|c|c|}
\hline Technologies & $\begin{array}{l}\text { Rendement } \\
\text { grain (kg/ha) }\end{array}$ & $\begin{array}{l}\text { Rendement } \\
\text { paille }(\mathrm{kg} / \mathrm{ha})\end{array}$ & $\begin{array}{l}\text { Nombre de } \\
\text { panicules } \\
\text { (nombre/ha) }\end{array}$ & $\begin{array}{l}\text { Rendement } \\
\text { panicules } \\
\text { (kg/ha) }\end{array}$ & $\begin{array}{l}\text { Poids de } \\
1000 \text { (g) }\end{array}$ \\
\hline $\begin{array}{l}\text { Fertilisation } \\
\text { T1F } \\
\text { T2F } \\
\text { T3F } \\
\text { T4F } \\
\text { T1F0 } \\
\text { T2F0 } \\
\text { T3F0 } \\
\text { T4F0 } \\
\text { Probabilité } \\
\text { Signification } \\
\text { Interaction trempage * } \\
\text { fertilisation } \\
\text { Signification } \\
\text { Moyenne générale } \\
\text { Ecart-type } \\
\text { CV\% }\end{array}$ & $\begin{array}{c}579,7 \mathrm{c} \\
685,9 \mathrm{~b} \\
768,2 \mathrm{ab} \\
889,1 \mathrm{a} \\
111,9 \mathrm{~d} \\
164,7 \mathrm{~d} \\
220,8 \mathrm{~d} \\
312 \mathrm{~d} \\
<0,001 \\
\mathrm{HS} \\
0,58 \mathrm{a} \\
\mathrm{NS} \\
467 \\
22 \\
28,7\end{array}$ & $\begin{array}{c}1289 c \\
1675 b \\
1996 \mathrm{~b} \\
2470 a \\
178 d \\
387 d \\
579 d \\
784 d \\
<0,001 \\
\mathrm{HS} \\
0,95 \mathrm{a} \\
\mathrm{NS} \\
1170 \\
34,21 \\
45\end{array}$ & $\begin{array}{l}26205 \mathrm{c} \\
30581 \mathrm{~b} \\
35778 \mathrm{~b} \\
40803 \mathrm{a} \\
10183 \mathrm{~d} \\
14193 \mathrm{~d} \\
17453 \mathrm{~d} \\
20538 \mathrm{c} \\
<0,001 \\
\mathrm{HS} \\
0,51 \mathrm{a} \\
\mathrm{NS} \\
24467 \\
156,42 \\
40\end{array}$ & $\begin{array}{c}773 \mathrm{~b} \\
972 \mathrm{~b} \\
1135 \mathrm{a} \\
1323 \mathrm{a} \\
144 \mathrm{c} \\
274 \mathrm{c} \\
341 \mathrm{c} \\
494 \mathrm{c} \\
<0,001 \\
\mathrm{HS} \\
0,49 \mathrm{a} \\
\mathrm{NS} \\
682 \\
26,12 \\
38,1\end{array}$ & $\begin{array}{c}21,67 \mathrm{~b} \\
22,5 \mathrm{~b} \\
24,61 \mathrm{a} \\
28,06 \mathrm{a} \\
17,94 \mathrm{c} \\
19,06 \mathrm{c} \\
19,86 \mathrm{c} \\
20,39 \mathrm{~b} \\
<0,001 \\
\mathrm{HS} \\
0,39 \mathrm{a} \\
\\
\mathrm{NS} \\
21,76 \\
4,66 \\
12,4\end{array}$ \\
\hline
\end{tabular}

Trp = trempage de semences, TTrp = témoin du trempage de semences (pratique paysanne de trempage), NS = non significatif, $\mathrm{HS}=$ hautement significatif, $\mathrm{F}=$ fertilisation de la parcelle de trempage de semences, $\mathrm{F} 0=$ fertilisation du témoin

\section{DISCUSSION}

Caractérisation physico-chimique des sols: Les sols des sites d'étude de Kolokani contenaient beaucoup de limons comparativement à ceux de Diéma (tableau 1). La teneur en sable était de $90 \%$ à Kolokani et environ $76 \%$ à Diéma (tableaux 1 et 2). Néanmoins, la teneur en argile variait peu entre les villages avec une valeur d'environ $2 \%$ (tableaux 1 et 2). Le pH (eau) des sites d'étude était généralement légèrement acide parfois moyennement acide selon les champs des paysans. La teneur en calcium sur les différents sites expérimentaux double celle du magnésium. Cependant, en considérant les autres propriétés physico-chimiques, tous les sites avaient une faible teneur en phosphore. La capacité d'échange cationique (CEC) y était moyenne. Tous les sols étaient pauvres en matière organique, en azote et en potassium (tableaux 1 et 2). Ces résultats corroborent avec ceux rapportés par Diakité et al (2018) et Traoré et al (2017) au Mali.

Effet de la technologie du trempage des semences avec différentes doses de fertilisation sur les rendements du sorgho: Le trempage des semences a permis une augmentation significative $(p<0,001)$ des rendements grain, paille, nombre et rendement panicules par rapport à la pratique paysanne. Le trempage a augmenté le poids de 1000 graines de $25 \%$ comparativement au témoin. II a amélioré les rendements grain, paille, nombre de panicules et poids panicules du sorgho de plus $100 \%$ par rapport à la pratique paysanne. Cette situation est en accord avec les résultats rapportés par d'autres auteurs (Harris, 2000 ; Coulibaly et al., 2010 ; Harris et al., 2006). Les rendements grain de toutes les doses de fertilisation étaient statistiquement différents ( $p<0,001)$. L'apport du fumier et de l'engrais minéral en micro-dose a augmenté significativement le rendement grain par rapport au témoin. Cette supériorité était en moyenne de $33 \%$. Ces types de fertilisants améliorent aussi le rendement paille de manière significative avec une supériorité de $54 \%$ par rapport au témoin. Ces résultats vont dans le même sens que ceux rapportés par 
ICRISAT (2009), Ibro et al (2012), Twomlow et al

\section{CONCLUSION}

La technologie de trempage des semences a donné des rendements grain de $731 \mathrm{~kg} / \mathrm{ha}, 1858 \mathrm{~kg} / \mathrm{ha}$ de paille, 33342 panicules/ha, $1051 \mathrm{~kg} / \mathrm{ha}$ de poids panicules; de loin supérieurs à ceux de la pratique paysanne de $+261 \%, 285 \%, 114 \%$ et $236 \%$ respectivement. Le trempage des semences a également un effet positif sur le poids de 1000 graines expliquant en partie la supériorité de rendement par un meilleur remplissage des grains en nutriments. Le trempage pourrait intéresser la vulgarisation agricole
(2010).

dans un contexte de changement climatique où l'installation des cultures est globalement gagée de récolte et très souvent de meilleur rendement.

Application des résultats: La technologie du trempage des semences permet de doubler voire tripler le rendement du sorgho par rapport à la pratique paysanne. Cette technologie peut être reproduite partout au Sahel et dans d'autres zones à écologie similaire.

\section{REMERCIEMENTS}

Nous remercions vivement World Vision pour l'appui financier dans la réalisation de la présente recherche.

\section{REFERENCES CITEES}

Abdoulaye Ibro, Mohamed Nasser Baco, Badiori Ouattara, Diakalia Sogodogo, Sabiou Mahamane, P.B. Irénikatché Akponikpè, Derek Peak, Anthony Kimaro et Saidou Koala, 2012. Le microdosage des engrais, une innovation qui profite aux femmes du Sahel.

Bougne, S., Job, C. and Job, D. (2000). Sugarbeet seed priming: solublization of the baic subunit of $11-\mathrm{S}$ globulin in individual seeds, Seed Science Research, 10: 153 -161.

Biasutti, M., and Sobel, A. H. (2009). Delayed Sahel rainfall and global seasonal cycle in a warmer climate. Geophysical Research. Letter, 36, L23707.

Coulibaly A., Aune J.B., Sissoko P., 2010. Etablissement des cultures vivrières dans les zones sahéliennes et soudano sahélienne du Mali. Rapport no. 60. Groupe de coordination des zones arides c/o Miljøhuset G9. 65 p. [Online] Available : http://www.drylandsgroup.org/Articles/1933.html?|=fr (octobre, 2018).

Damirkaya, M., Okgu, G., Atak, G. Y. and Kolsarici, O. (2006). Seed treatments to overcome salt and drought stress during germination in sunflower (Helianthus annus L.). European Journal of Agronomy, 24: 291-295.

Harris, D., Tripathi, R. S. and Joshi, A. (2000). On- farm priming to improve crop establishment and yield in direct- seeded rice in IRRI: International Workshop on Dry -seeded Rice Technology`. Held in Bangkok, $25-28$ January 2000. The international Rice
Research institute, Manila, The Philippines, 164. Harris, D., Pathan, A. K., Gothkar, P., Joshi, A., Chivasa, W. and Nyamudeza, P. (2001). On-farm seed priming using participatory methods to revive and refine a key technology. Agricultural Systems, 69:151164.

ICRISAT (2009). Fertilizer Microdosing, Boosting production in unproductive lands. ICRISATPatancheru.

Jones, M. J. and Wanbi, A. (1992). Site factor influence on barley response to fertilizer in on farm trials in northern Syria: descriptive and predictive models, Experimental Agriculture, 28:63-87.

Kropff, M. J. and Van laar, H. H. (1993). Modeling cropweed interactions. $\mathrm{CAB}$ international Wallingford, U.K., 272.

Traore K., Sidibé D.K., Coulibaly H., Bayala J. 2017b. Optimizing yield of improved varieties of millet and sorghum under highly variable rainfall conditions using contour ridges in Cinzana, Mali. Agriculture and Food Security, 6:11. DOI 10.1186/s40066-016-0086-0.

Twomlow, S., Rohrbach, D., Dimes, J., Rusike, J., Mupangwa, W., Ncube, B., Hove, L., Moyo, M., Mashingaidze, N., \& Mahposa, P. (2010). Micro-dosing as a pathway to Africa's Green Revolution: evidence from broad-scale on-farm trials. Nutrient Cycling in Agroecosystems, 88.3. 